\title{
Baouît (2019)
}

\section{Gisèle Hadji-Minaglou}

\section{OpenEdition \\ Journals}

Édition électronique

URL : https://journals.openedition.org/baefe/880

DOI : $10.4000 /$ baefe. 880

ISSN : 2732-687X

Éditeur

ResEFE

\section{Référence électronique}

Gisèle Hadji-Minaglou, « Baouît (2019) » [notice archéologique], Bulletin archéologique des Écoles

françaises à l'étranger [En ligne], Égypte, mis en ligne le 05 octobre 2020, consulté le 28 juillet 2021

URL : http://journals.openedition.org/baefe/880 ; DOI : https://doi.org/10.4000/baefe.880

Ce document a été généré automatiquement le 28 juillet 2021.

\section{(c) $($ i) $(9)$}

Le Bulletin archéologique des Écoles françaises à l'étranger est mise à disposition selon les termes de la Licence Creative Commons Attribution - Pas d'Utilisation Commerciale - Pas de Modification 4.0

International. 


\title{
Baouît (2019)
}

\author{
Gisèle Hadji-Minaglou
}

\section{NOTE DE L'AUTEUR}

Année de la campagne : 2019 (18 mars - 21 avril)

Numéro et intitulé de l'opération de terrain : 17126 - Fouilles du monastère de Baouit, Moyenne Égypte

Composition de l'équipe de terrain : L'équipe était composée de Gisèle Hadji-

Minaglou (archéologue-architecte, Ifao), Florence Calament (coptologue, épigraphiste, musée du Louvre), Cédric Meurice (coptologue, musée du Louvre), Sayed Awad Mohamed Sheuib (archéologue, MoTA), Eleni Efthymiou (archéologue, ministère grec de la culture), Catherine Jolivet (historienne de l'art, EPHE), Héléna Rochard (historienne de l'art, EPHE), Delphine Dixneuf (céramologue, CNRS, UMR 7298 LA3M), Alexandra Konstantinidou (céramologue, ministère grec de la culture), Anna Południkiewicz (céramologue, université de Varsovie), Paul Bailet (anthropologue, Dracénie Provence Verdon agglomération, unité archéologie et patrimoine), Alanna Troconis-Noblet (architecte), Alice Cétout (dessinatrice, Inrap), Abdullah Ali Harb (restaurateur, MoA), Joanna Kania, Bruno Szktonicki (restaurateurs) et Ihab Mohamed Ibrahim (photographe, Ifao). Suzan Abdel Imam, Ali Abdel Alim et Irini Philippe Aziz Guergues représentaient ministère du Tourisme et des Antiquités (MoTA). Cherif Gamal Abdel Kerim Ibrahim et Chaima Felfel Fathi ont participé aux travaux en tant que stagiaires.

Partenariat institutionnel : La mission bénéficie du soutien du musée du Louvre. Etablissements porteurs du projet : Musée du Louvre / IFAO

1 Le dégagement complet de l'église principale s'étant terminé en $2018^{1}$, les fouilles de cette année se sont essentiellement concentrées à l'extérieur de l'édifice, en particulier au sud de celui-ci, où un cimetière avait été découvert en $2016^{2}$. À l'intérieur, un sondage a été effectué dans la partie ouest du bêma actuel, afin d'y repérer un bêma plus ancien et de confirmer l'existence dans le sanctuaire de sépultures antérieures à l'église ${ }^{3}$. Dans le naos, le mur adossé à la première colonne de la nef latérale sud, en 
partant du bêma, qui avait vraisemblablement été construit en même temps que les piliers $^{4}$, a été démonté. Parallèlement, la restauration et l'étude des fragments de peinture provenant de la salle $7 \mathrm{du}$ bâtiment 1 , dans le secteur nord, se sont poursuivies en laboratoire.

\section{Le cimetière au sud de l'église}

2 Lorsqu'en 2016 l'on démonta une partie du mur sud de l'église principale, faite de remplois, il apparut que les blocs supérieurs, effondrés vers l'extérieur ${ }^{5}$, recouvraient des sépultures. Le démontage du mur nécessita que l'on fouille tant à l'intérieur qu'à l'extérieur de l'édifice, jusqu'à ce que soit atteinte une base solide sur laquelle appuyer un nouveau mur 6 . Ainsi, les sépultures n'ont été fouillées que sur une faible profondeur et uniquement le long du mur. 24 d'entre elles ont été mises au jour : 10 corps étaient ensevelis contre le mur ou à peu de distance de celui-ci et 14 l'étaient dans un petit espace de 2,60 × 1,50 m7 . En 2017, 10 sépultures supplémentaires ont été mises au jour plus à l'ouest, le long du mur8.

3 En 2019, la fouille du cimetière débuta sous le petit espace, une fois déposés, pour des raisons de sécurité, les murets le délimitant; elle s'étendit vers le sud, dans le but de trouver la bordure de l'enclos funéraire dans cette direction, les deux limites est et ouest étant déjà connues. Elle s'étendit également vers l'est, afin de dégager l'entrée sud de l'église. Le mur sud du cimetière n'a pas été atteint, malgré une extension de $10 \mathrm{~m}$, la surface actuellement couverte étant de $10 \times 19,50 \mathrm{~m}$ (fig. 1). Quoi qu'il en soit, cette surface étant très importante, nos investigations ont été menées, à partir du niveau supérieur des inhumations, jusqu'à une distance de $4 \mathrm{~m}$ du mur de l'église et $3 \mathrm{~m}$ du mur ouest; en outre, le rocher n'a été atteint que sous le petit espace et devant l'entrée de l'église. En dépit de ces restrictions, 30 individus ont été étudiés ; 28 étaient enterrés à l'intérieur de l'enclos funéraire et 2 devant l'entrée de l'église; d'autres individus ont été repérés, mais ont été laissés sur place pour la prochaine campagne. 
Fig. 1. Vue générale de la zone fouillée en 2019 (Ihab Mohamed Ibrahim).

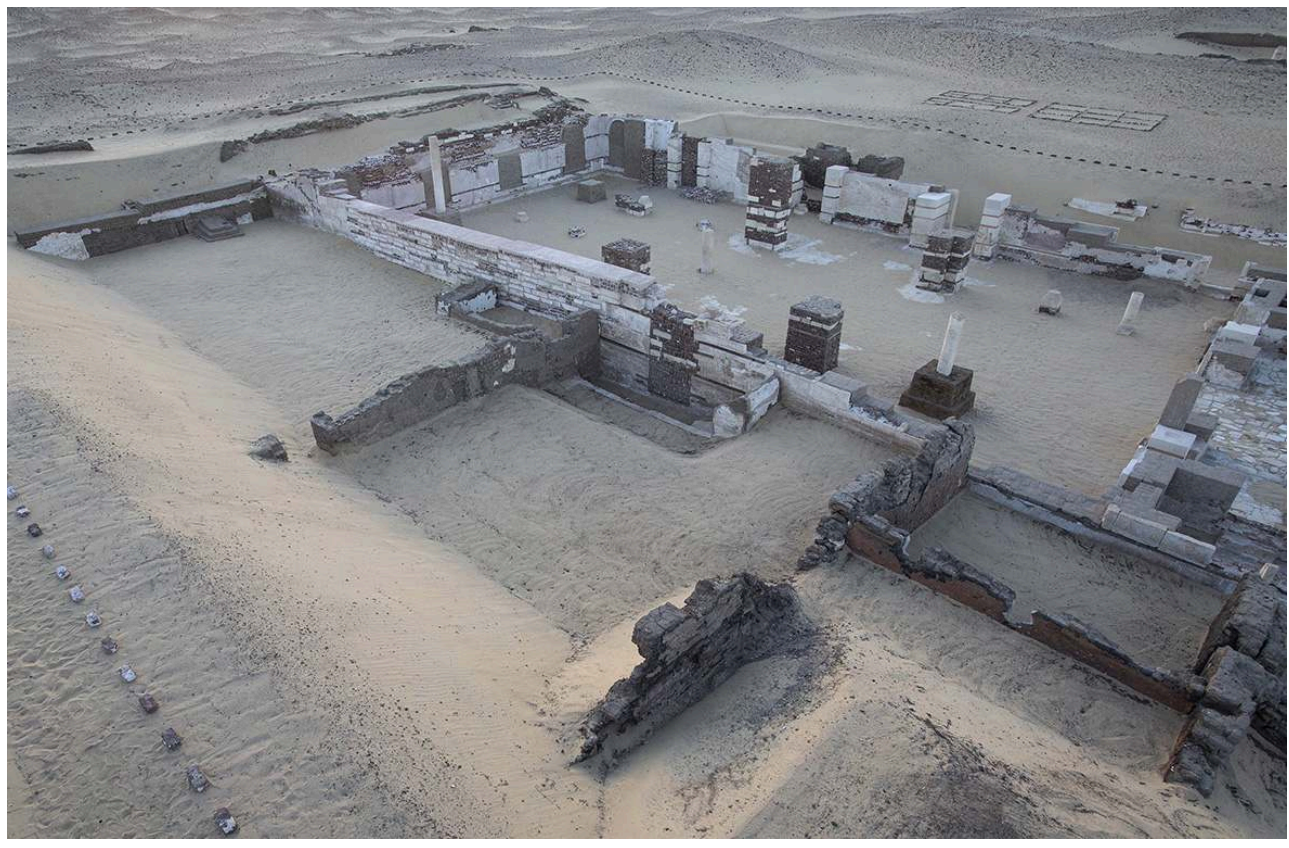

(c) Ifao/Louvre. 17126_2019_NDMPF_001

Fig. 2. Inhumations du niveau supérieur (B41, 42 et 43) (Sayed Awad Mohamed Sheuib).

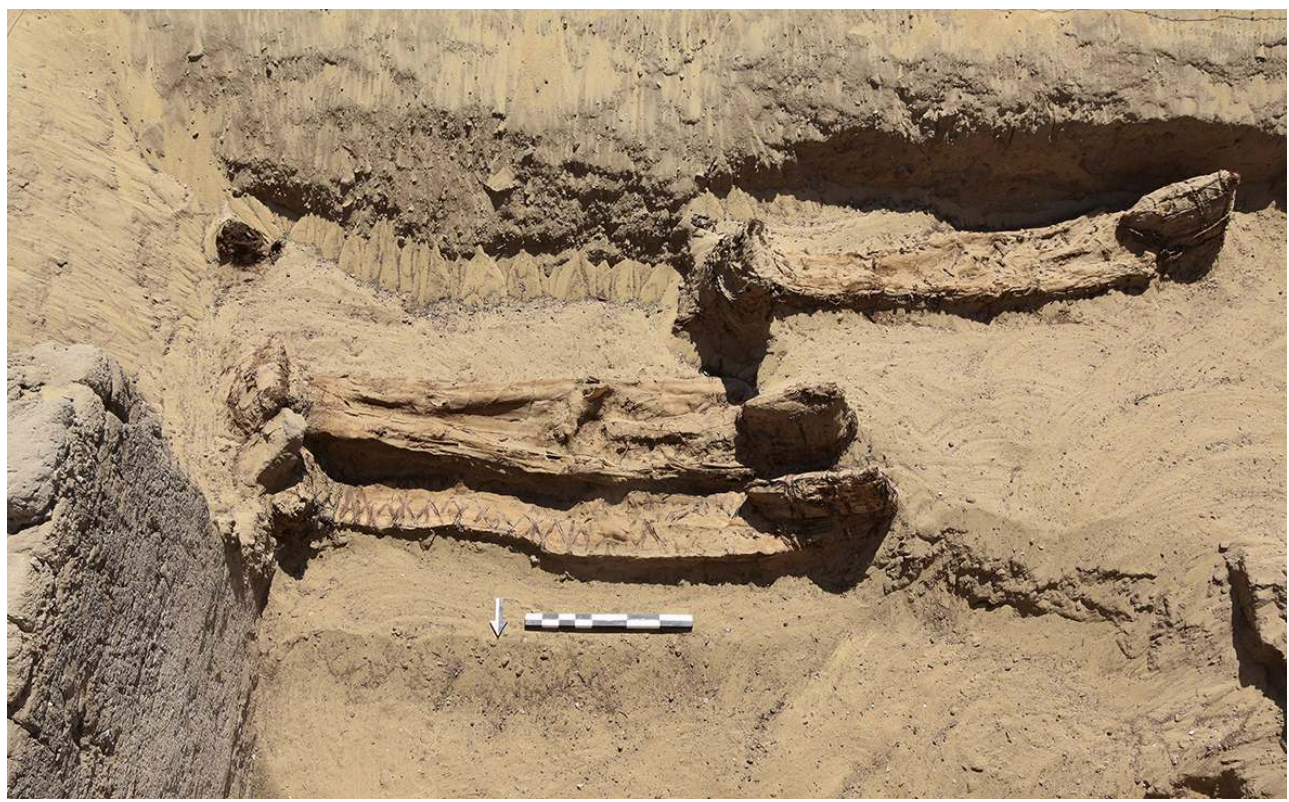

(c) Ifao/Louvre. 17126_2019_NDMPF_002

Trois niveaux d'inhumation ont été identifiés. Au niveau supérieur (fig. 2), les corps étaient inhumés directement dans la terre et leurs vêtements et linceuls sont bien conservés. Comme ceux découverts les années précédentes, ils se caractérisent par des protubérances faites de fibres végétales, qui enveloppent les pieds et la tête et sont maintenues par des nervures de palmier, créant ainsi une forme nette et pointue 9 Entre les jambes de l'une de ces dépouilles était placée une pyxide en bois ${ }^{10}$. Le niveau intermédiaire, uniquement fouillé sous le petit espace, comptait 8 individus, également 
enterrés dans la terre. Bien qu'ils soient moins bien conservés, nous avons néanmoins constaté que leur tête et leurs pieds étaient également enveloppés dans des fibres de palmier, les protubérances étant toutefois moins hautes et moins sophistiquées. Ces deux niveaux sont contemporains de la dernière phase d'occupation de l'église, à savoir le $\mathrm{VIIII}^{\mathrm{e}} \mathrm{s}$. et le début $\mathrm{du} \mathrm{IX}^{\mathrm{e}} \mathrm{s}$. Le troisième niveau de sépultures est également contemporain de l'église, mais semble remonter au début de son utilisation, c'est-à-dire au $\mathrm{VII}^{\mathrm{e}} \mathrm{s}$. 7 dépouilles ont été mises au jour sous le petit espace et 2 devant l'entrée sud de l'église ; elles étaient placées dans la partie supérieure de caissons, dont certaines d'entre elles ont sectionné les parois. Ces caissons sont antérieurs à l'église et contemporains de ceux découverts en 2009 sous l'escalier du sanctuaire et dans le naos ${ }^{11}$. Comme ces derniers, ils sont délimités par des murets en briques crues, qui reposent sur le substrat naturel et délimitent des espaces rectangulaires, aux dimensions adéquates pour recevoir des dépouilles humaines (fig. 3). Les caissons mis au jour cette année ne semblent pas avoir été utilisés au moment de leur construction car, contrairement à ceux trouvés à l'intérieur de l'église, ils n'avaient aucune couverture.

5 Les deux banquettes laissées en place en 2016 et $2017^{12}$ étaient adossées au mur ouest du cimetière et reposaient sur la couche renfermant les sépultures les plus récentes. Entièrement construites en briques crues et recouvertes d'un enduit d'argile lissé, elles mesuraient $2 \times 1,40 \mathrm{~m}$ pour celle située au nord et 2,07 $\times 1,40 \mathrm{~m}$ pour celle située au sud. Leur dépose révéla l'existence d'un monument funéraire situé juste au-dessous de la banquette sud. Ce monument se présente comme un podium rectangulaire à trois gradins, en briques crues recouvertes, comme les banquettes, d'un enduit d'argile lissé (fig. 4). Ses dimensions sont $1,90 \times 1,50 \mathrm{~m}$, compte non tenu de sa base arrondie, qui forme un gradin supplémentaire côté sud et déborde côté est. Cette base, cassée sur son côté nord par une inhumation, fait apparaître un second monument en briques sousjacent. 
Fig. 3. Tombes à caissons (Sayed Awad Mohamed Sheuib).

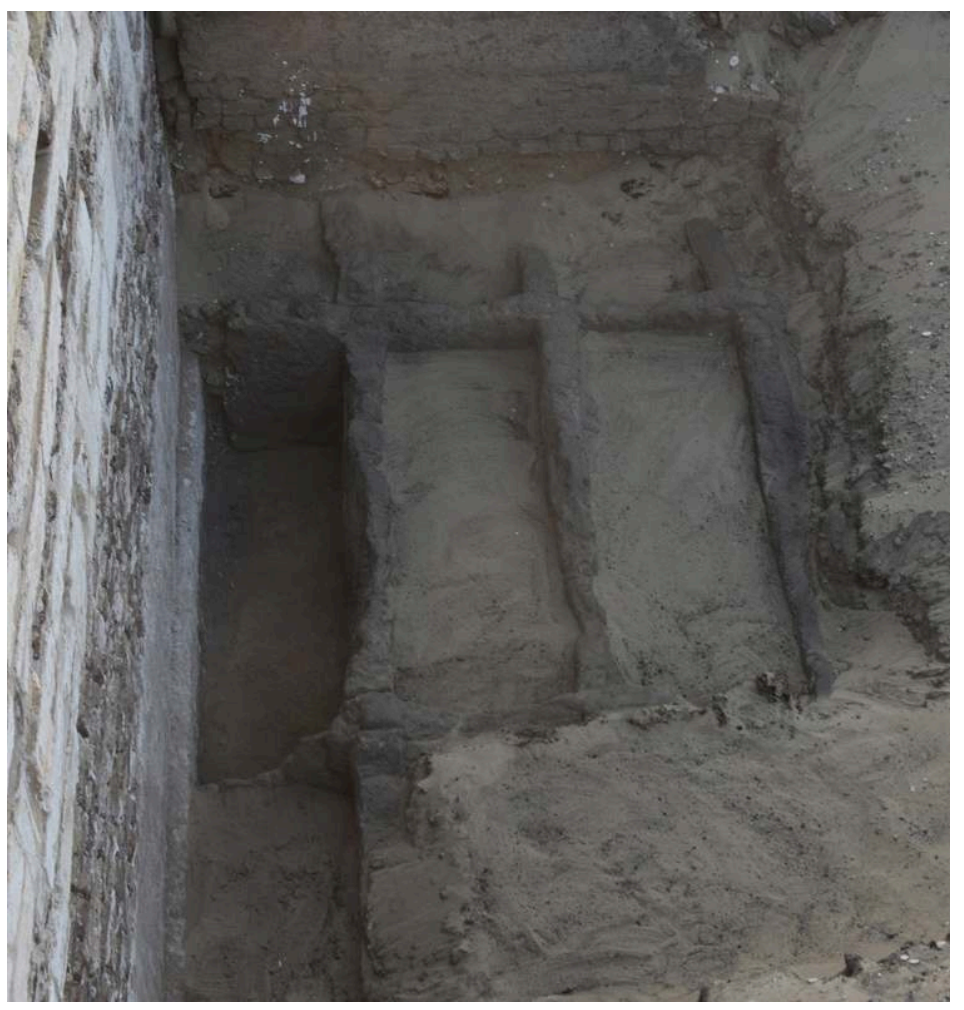

(C) Ifao/Louvre. 17126_2019_NDMPF_003

Fig. 4. Inhumations, stèles et monument funéraire contre le mur ouest du cimetière (G. HadjiMinaglou).

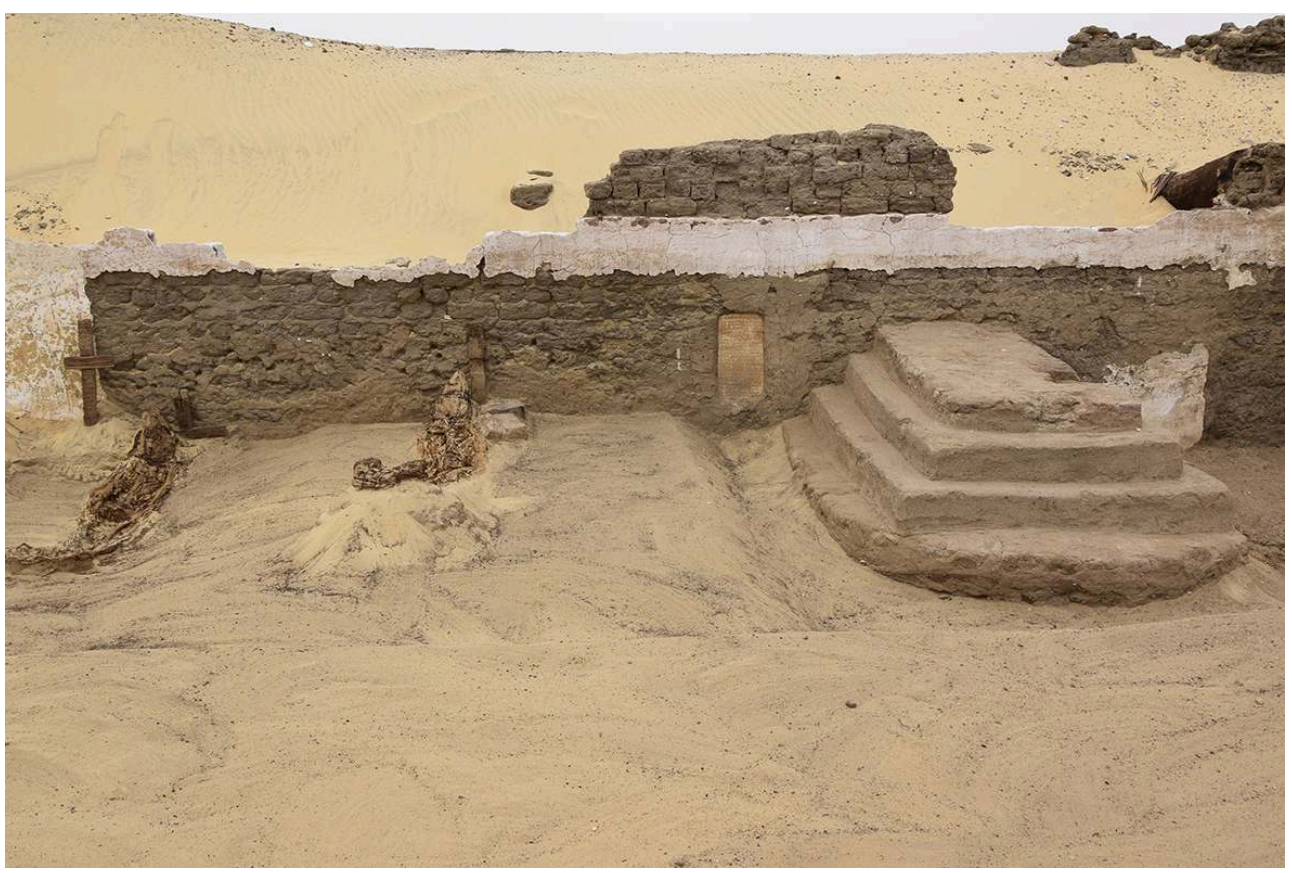

(C) Ifao/Louvre. 17126_2019_NDMPF_004

6 Plusieurs stèles funéraires ont été trouvées in situ (fig. 4), qui donnent toutes des datations relatives, par indiction. Une stèle en pierre calcaire gravée était encastrée 
dans le mur ouest du cimetière, immédiatement au sud du monument à gradins. Elle concerne un certain Apa Georges. Une fois descellée, il apparut qu'elle est également écrite au verso, où le même texte inachevé est peint en rouge et partiellement gravé. Trois autres stèles, des croix en bois, étaient enfoncées dans le sol, contre le mur. La première de ces croix en partant $d u$ monument funéraire a perdu sa traverse et l'inscription qu'elle porte sur son montant est très mal gravée et illisible. La deuxième est en revanche complète, incisée avec soin, et concerne un dénommé frère Jean ; trois clous en fer, dont deux sont encore présents, permettaient de la maintenir contre la paroi. La troisième, quelques dizaines de centimètres plus au sud, présente une particularité : sa traverse n'est pas originale et a été placée à l'envers. Une demitraverse manquante a été retrouvée plus loin dans la terre. Les deux pièces de bois s'emboîtent parfaitement et le texte est cohérent, mais nous n'avons ni le nom du défunt, ni la date de son décès. Plusieurs autres fragments de stèles en bois ont été trouvés dans la terre autour et au-dessus des sépultures. Parmi eux, signalons le bas d'un montant de croix sur lequel des palmes étaient liées au moyen d'une corde (fig. 5). Des restes de croix en bois similaires ont été trouvés par Jean Clédat, dans la nécropole désertique au nord du kôm et dans la nécropole méridionale. ${ }^{13}$ Enfin, une stèle en calcaire complète, face contre terre dans l'angle du mur sud de l'église et du mur ouest du cimetière, était visiblement tombée de ce dernier : il s'agit de la stèle d'un dénommé Kosma.

7 Sur le versant est du mur est du cimetière, à côté de la stèle de Patermoute découverte et déposée en $2016^{14}$, a été repérée une inhumation contemporaine de celles du niveau supérieur, qui n'a pas été entièrement dégagée ${ }^{15}$. Sur la tête de la personne inhumée était posée une couronne en fibres végétales et tissus (fig. 6) : des fibres de papyrus et des bandes de tissu de couleur sont enroulées autour de trois tiges formant un cercle d'épaisseur dégressive. À ma connaissance, aucune couronne de ce type n'a été trouvée ailleurs, au-dessus d'une sépulture chrétienne. Signalons toutefois que dans le cimetière de Fag el-Gamous, dans le Fayoum, une corde circulaire, faite de fils de laine rouge, que les fouilleurs ont interprétée comme l'anse d'une croix ankh, était posée sur le visage de la dépouille d'une femme ${ }^{16}$. À Qarara, une guirlande de feuilles de palmier tressées était passée autour du cou de deux dépouilles ${ }^{17}$. Des guirlandes similaires ont été signalées à Licht, où elles entouraient le cou ou les chevilles des individus ${ }^{18}$. 
Fig. 5. Montant de croix avec palmes (Ihab Mohamed Ibrahim).

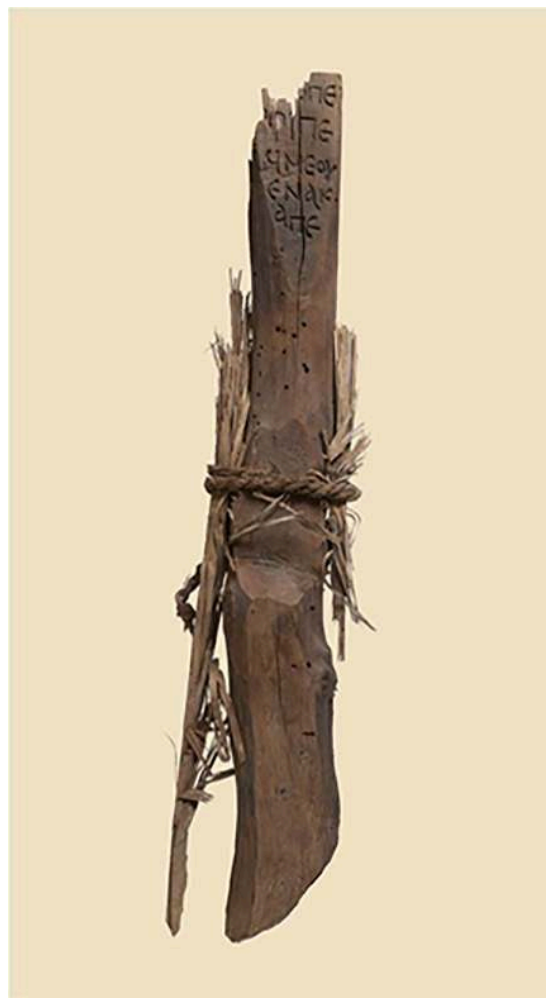

(c) Ifao/Louvre. 17126_2019_NDMPM_001

Fig. 6. Couronne (G. Hadji-Minaglou).

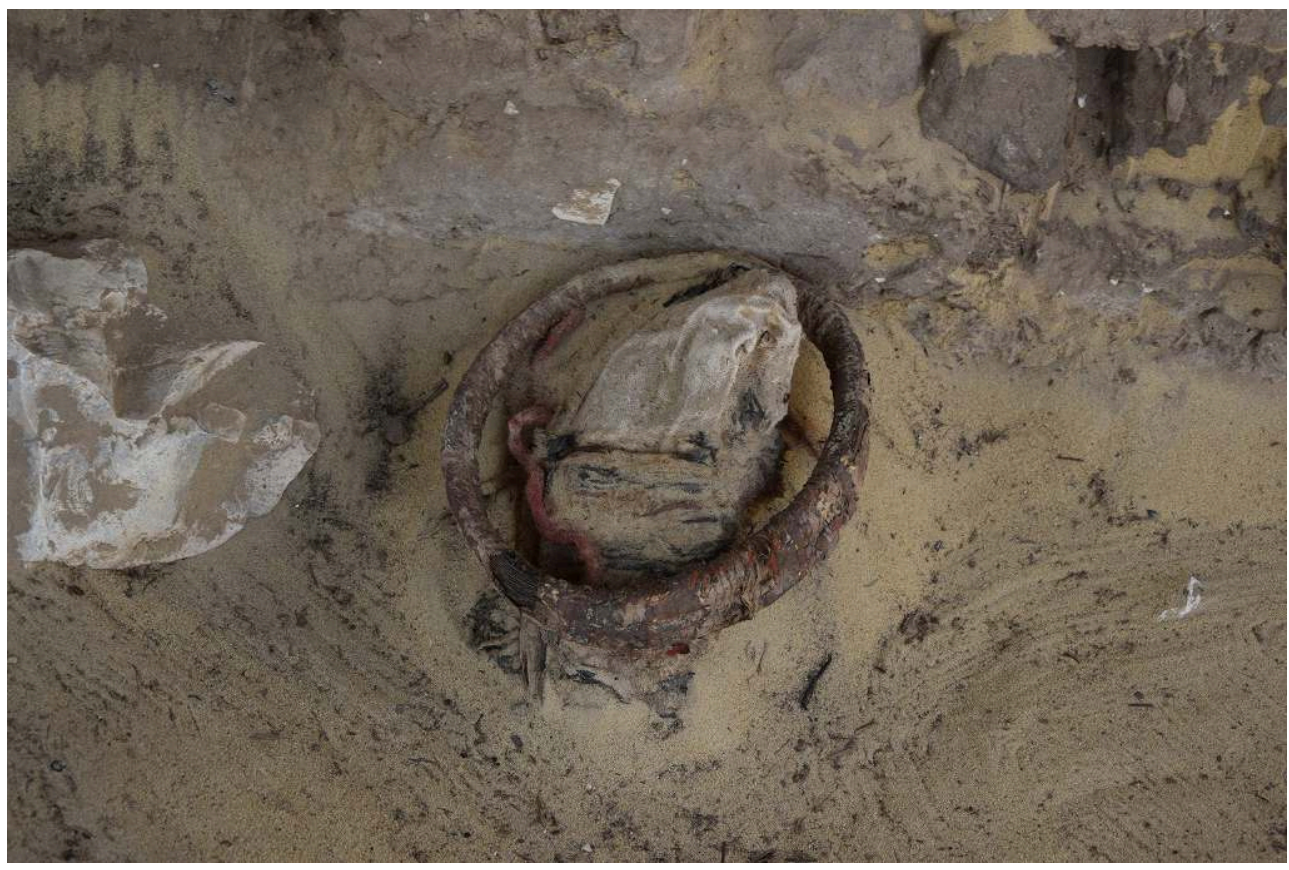

(c) Ifao/Louvre. 17126_2019_NDMPF_005 


\section{L'entrée sud de l'église}

(fig. 7)

8 La présence de sépultures devant l'entrée sud de l'église montre que le cimetière s'étendait jusqu'à cet endroit et qu'un second enclos cémétérial existait à l'est de celui déjà connu. Les deux enclos étaient contemporains et deux passages étaient ménagés entre eux, dans leur mur commun. Dans la dernière phase d'occupation du cimetière, celles des VIII et IX ${ }^{e}$ s., l'entrée de l'église était d'évidence bouchée.

Devant l'entrée bouchée se trouve un espace oblong de 5,45 ×1,20 m, ouvert au sud, limité par le mur de l'enclos à l'ouest et par un mur en briques avec un retour vers le sud, à l'est. Cet espace n'était pas couvert, ainsi que le montre le sommet du mur est, dont l'enduit est encore conservé. Ce mur repose sur une marche, sur laquelle est fixée une croix en plomb ornée en son centre d'un cabochon. Devant la marche, sur le sol en terre, se trouve une dalle rectangulaire $(1,42 \times 0,58 \mathrm{~m})$, orientée est-ouest. Inscrite, elle était partiellement recouverte d'enduit et érodée sur les deux tiers de sa surface. Une croix latine est gravée en son centre et le texte est divisé en quatre sections. Du fait des lacunes importantes, le sens de lecture et l'identification du texte sont rendus difficiles.

Le mur est, son retour et le mur de l'église étaient ornés de peintures. Celles du mur de l'église sont très effacées. En revanche, les autres sont relativement bien conservées, bien qu'extrêmement fragiles. Le décor du mur est (fig. 8) est divisé en deux registres : au registre supérieur trônent la Vierge et l'Enfant, flanqués des archanges Michel et Gabriel en costume impérial; au registre inférieur, quatre saints désignés comme "notre père » sont assis sur un banc gemmé identique au trône de la Vierge. Ils sont tous vêtus à l'antique et tiennent un codex orfévré de leur main gauche voilée tandis que leur main droite est posée sur la tranche. On retrouve les deux registres sur le retour sud; chacun d'eux est occupé par deux saints assis sur un banc gemmé, selon la même iconographie. L'un d'eux, sur le registre inférieur, a pu être identifié grâce à sa légende : « Notre père apa Pamoun, l'homme (de) Hnès ». Apa Pamoun de Hnès est bien attesté à Baouît, notamment dans la salle 7, ainsi que dans l'église nord. Sur le mur de l'église, on distingue la présence d'une nouvelle série de saints appartenant au même ensemble. Ce petit espace concentre ainsi pas moins de quatorze saints assis sur des bancs gemmés. 
Fig. 7. Entrée sud de l'église (Sayed Awad Mohamed Sheuib).

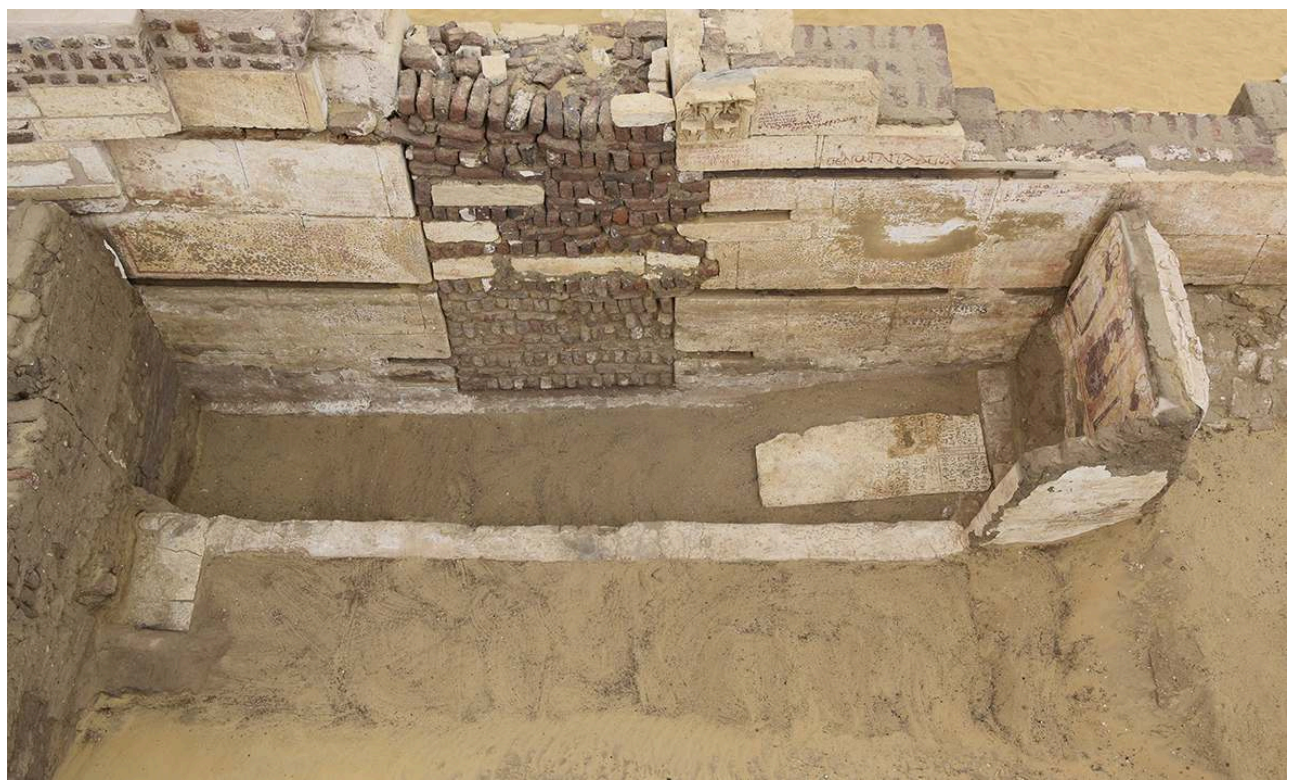

(c) Ifao/Louvre. 17126_2019_NDMPF_006

Fig. 8. Mur est de l'espace devant l'entrée sud de l'église (Ihab Mohamed Ibrahim).

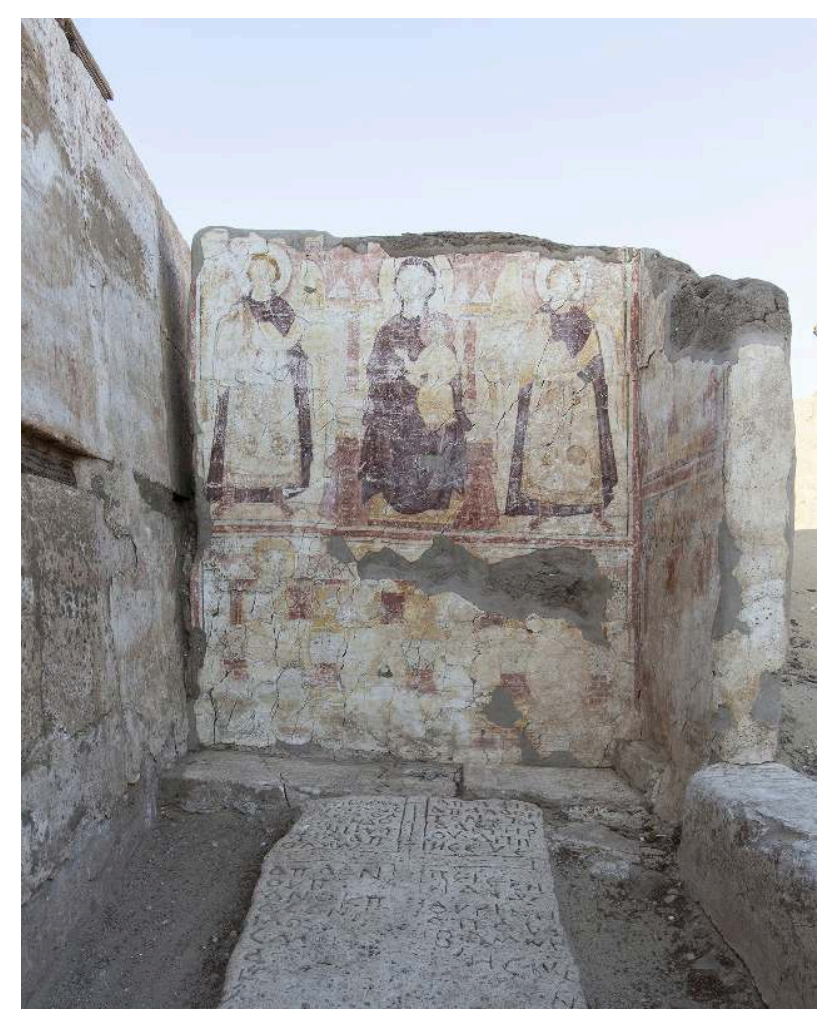

(C) Ifao/Louvre. 17126_2019_NDMPF_007 


\section{Les travaux à l'intérieur de l'église}

11 Un sondage a été effectué sur une surface de $13 \mathrm{~m}^{2}$ dans la moitié ouest du bêma, plus précisément devant l'ouverture sur le naos (fig. 9). À cet endroit, le dallage, fait de plaques de calcaire rectangulaires dans la continuité du naos, avait été retiré et remplacé par des dalles cassées et des fragments de briques cuites ${ }^{19}$. Ces éléments fragmentaires étaient placés sur une couche de sable propre préalablement étalé sur un remblai sablonneux. Ce remblai, qui a pu être daté du IXe siècle, recouvrait des éléments attendus - les restes d'un bêma antérieur et des caissons en brique crue, identiques à ceux des sépultures situées sous l'escalier du sanctuaire, dans la nef sud du naos ${ }^{20}$ et dans le cimetière sud, décrit plus haut-, mais aussi deux énormes jarres, tout à fait inattendues. La présence de ces jarres est déroutante et n'a pour l'instant pas reçu d'explication. La seule certitude est qu'elles sont contemporaines de la première phase du bêma. L'une, au nord, était emplie de sable et de pierres, dépourvue de fond, fichée dans le substrat rocheux; l'autre était complète et emplie de sable propre. Chacune a sa lèvre entourée d'un rebord composé, pour l'une, de briques cuites enduites de chaux (probablement remployées) et liées avec un mortier d'argile, pour l'autre, de ces mêmes éléments et de fragments de pierres. Toutes deux étaient installées dans des caissons, dont elles ont cassé les parois. Un caisson situé entre elles a été retrouvé coupé en deux par un muret. Sa moitié est était remplie de sable propre ; l'autre moitié n'a pas été fouillée, car elle passait sous le dallage de la nef. Le muret a visiblement été construit pour retenir le sable accumulé dans la moitié ouest. Au-dessus du caisson et en partie enfoncé dans le sable se trouvait un vase renversé. Une fois retourné, il s'est avéré contenir une boule de tissu ressemblant fort à un vêtement enroulé. Cette boule n'a pas encore été défaite et attend d'être examinée par R. Cortopassi. Outre ceux abîmés par les jarres, deux autres caissons ont été repérés, plus au nord. L'un était scellé par une couverture en briques enduites d'argile et formant un sol, couverture que nous n'avons pas déposée : il s'agit du même type de sépulture que celle du naos. L'autre n'avait ni couverture, ni dépouille à l'intérieur. Les sépultures continuent vers l'est et l'ouest, le bêma étant certainement construit au-dessus.

12 La limite du bêma le plus ancien a été repérée $80 \mathrm{~cm}$ à l'ouest du plus récent; il est précédé d'une marche de $25 \mathrm{~cm}$ de large. Haut de $20 \mathrm{~cm}$, le soubassement de cette marche repose directement sur les caissons; la plateforme du bêma se trouve $18 \mathrm{~cm}$ plus haut. Le rebord de l'une et l'autre est délimité par un alignement nord-sud de briques cuites et de pierres en calcaire, tandis que le reste de la maçonnerie est en briques cuites disposées perpendiculairement au rebord. L'ensemble est recouvert d'un mortier de chaux, qui a conservé la trace de dalles. Les mêmes traces se retrouvent, au même niveau, sous le bêma le plus récent à l'emplacement de l'autel ${ }^{21}$.

13 Dans la nef centrale du naos, un mur adossé à la première colonne en partant du bêma et appartenant à la dernière phase de l'église a été démonté (fig. 10). Il comptait 13 assises composées de fragments sculptés, de blocs de calcaire et de briques cuites. 227 blocs décorés ont ainsi été déposés; 89 d'entre eux ont été conservés en magasin, les autres ont été enterrés à proximité dans la nécropole ${ }^{22}$. Cinq groupes de décor se détachent parmi les blocs mis de côté : bases et chapiteaux de pilastre, éléments de frises d'acanthe, éléments de frises avec motifs de grecques, éléments de frises avec feuilles se développant autour de motifs centraux, éléments de frises avec feuilles d'acanthes et grappes de raisins placés en alternance et un élément de frise avec oves et tableaux. Des 
pièces en marbre ont également été trouvées: deux fragments et une base de colonnettes. Deux fragments à feuilles d'acanthe ont conservé des restes de peinture rouge. Une partie de ces sculptures provient de l'église elle-même et une autre des alentours immédiats. Quoi qu'il en soit, ces sculptures viennent s'ajouter à un corpus déjà riche et mettent en évidence, une fois de plus, les grandes similitudes existant entre le décor de l'église principale et celui de l'église sud.

Fig. 9. Sondage dans le bêma (Sayed Awad Mohamed Sheuib).

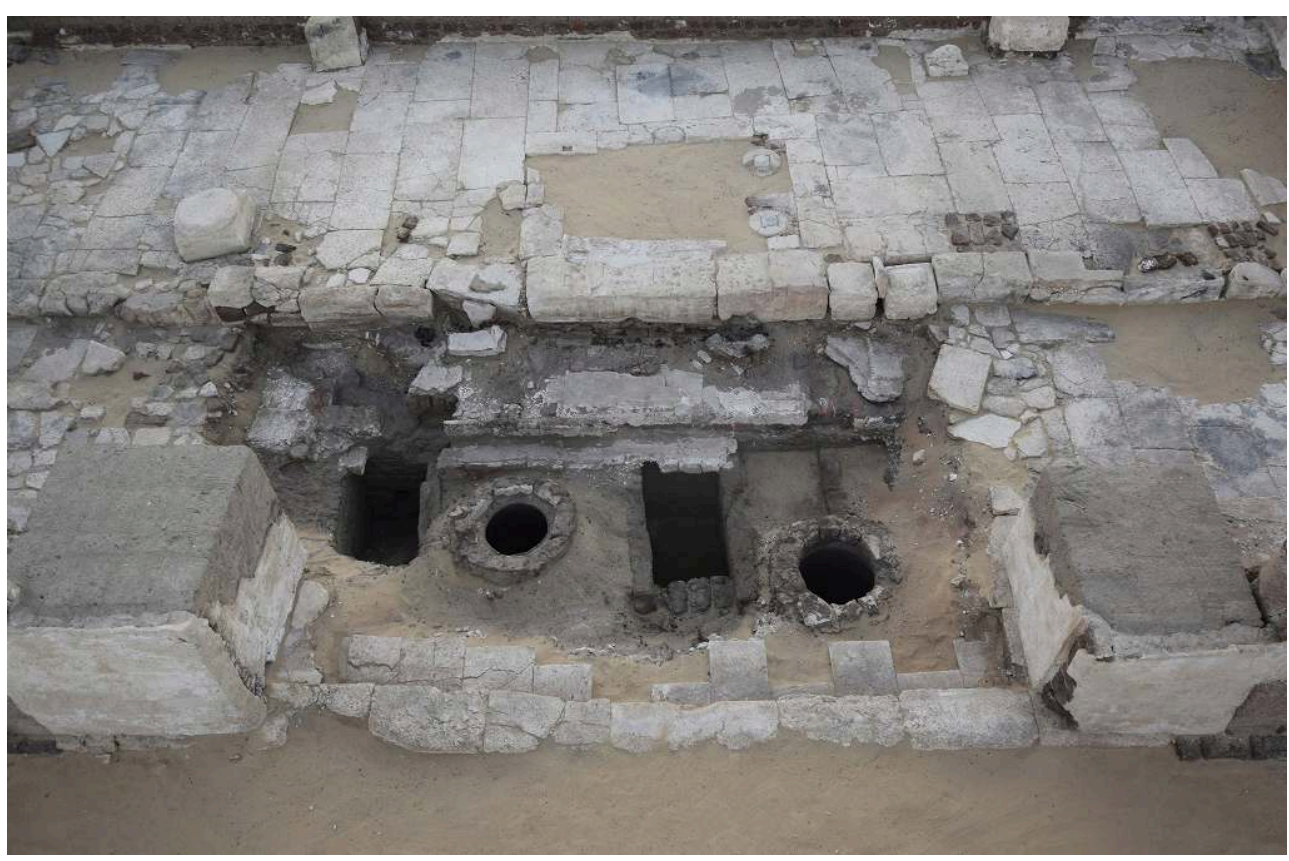

(c) Ifao/Louvre. 17126_2019_NDMPF_008 
Fig. 10. Mur de la nef centrale en cours de démontage (C. Meurice).

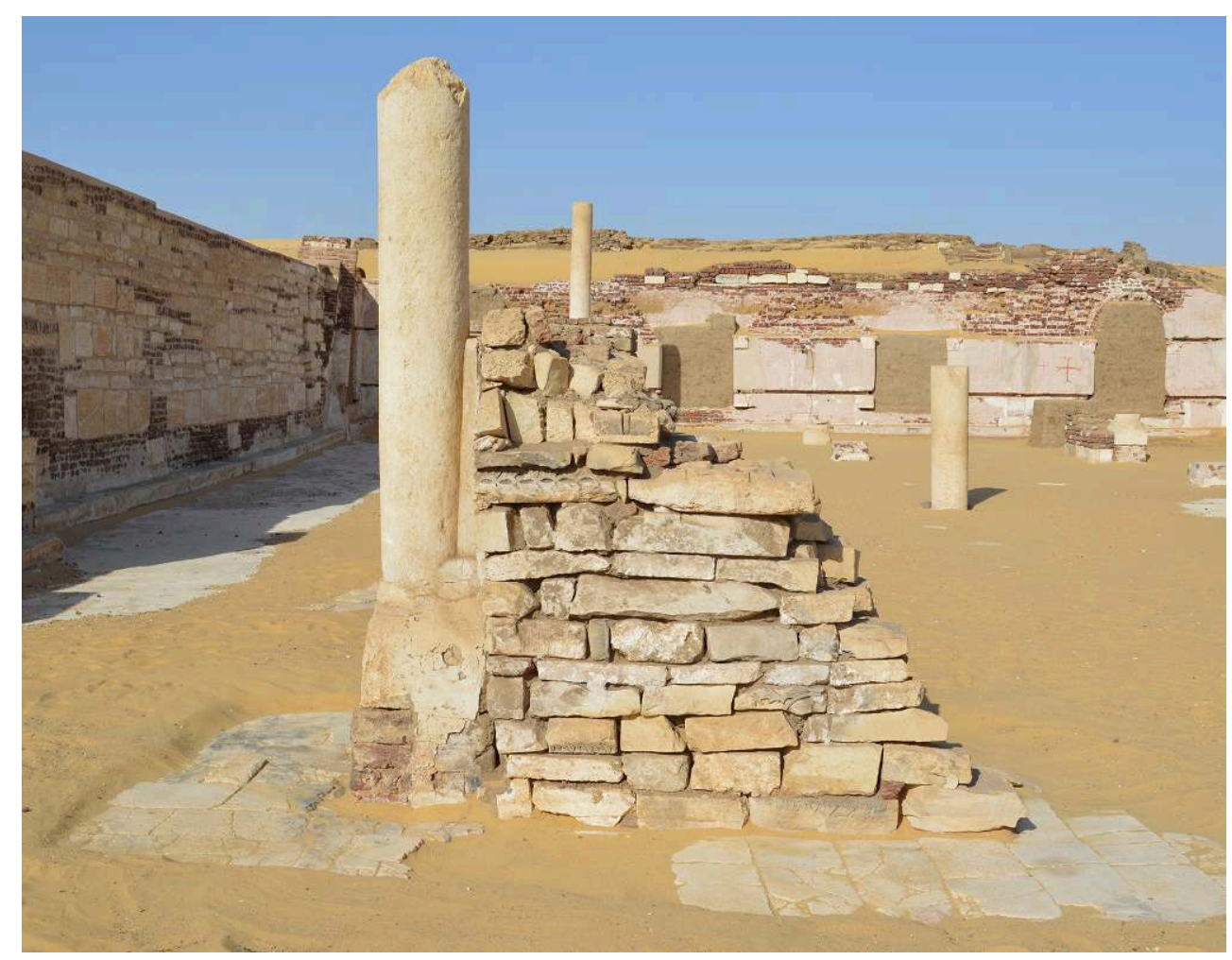

(c) Ifao/Louvre. 17126_2019_NDMPF_009

\section{La restauration des peintures de la salle 7}

En raison de l'inventaire général engagé par le MoTA, nous n'avons pu sortir du magasin qu'un nombre réduit de portoirs. Le travail de restitution des peintures s'est ainsi limité aux peintures de la niche centrale du mur est de la salle, amorcée en $2018^{23}$. En outre, ce travail s'est concentré sur l'image de la conque absidale, en particulier sur le Christ en majesté et les personnages se trouvant à sa droite (fig. 11). Le Christ, vêtu d'une tunique et d'un manteau pourpre, siège sur un trône gemmé agrémenté d'un épais coussin, au centre d'une gloire lumineuse. Il lève la main droite dans un geste de bénédiction et tient de l'autre le Livre ouvert sur lequel est inscrit le nom d'Emmanuel. L'auréole, marquée de cercles concentriques bleu ciel puis orange, se détache sur un fond bleu nuit parsemé d'étoiles où brille l'astre solaire. Deux des quatre créatures aux ailes constellées d'yeux qui symbolisent les quatre évangélistes (l'homme, le lion, l'aigle et le bœuf) et entourent le Christ ont été en partie remontées (l'homme et le lion), en même temps que l'archange se trouvant à la droite du Christ. La restitution du décor de la conque pose le problème de la hauteur du cul de four et de la courbure de la surface peinte, qu'il est difficile, étant donné le plan rectangulaire et les grandes dimensions de la base de l'abside $(1,35 \times 35 \mathrm{~cm})$, de reproduire avec précision. 
Fig. 11. Restitution partielle du décor de la niche centrale du mur est de la salle 7 (Ihab Mohamed Ibrahim).

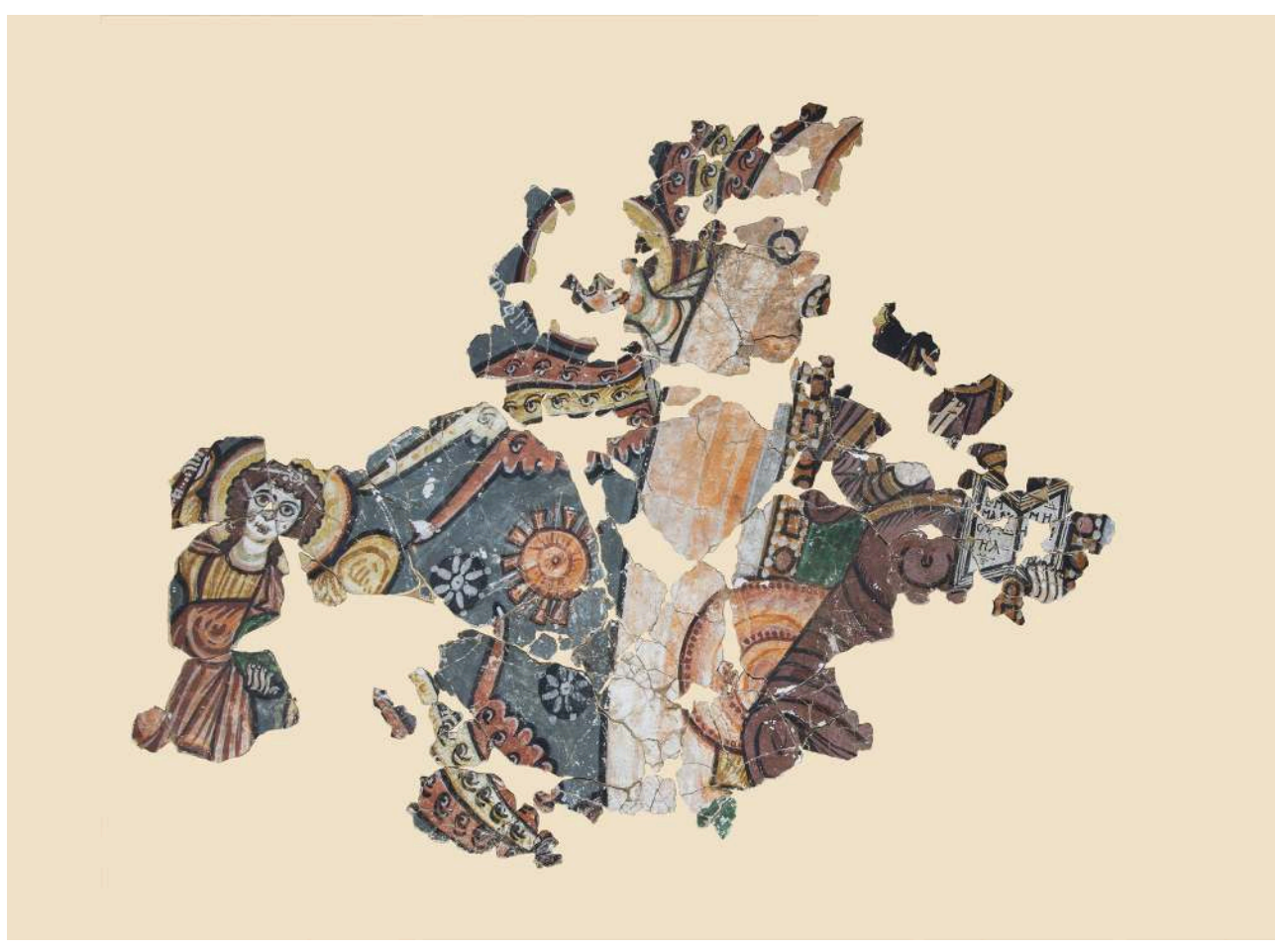

\section{BIBLIOGRAPHIE}

\section{CLÉDAT 1999}

Jean Clédat (†), Le monastère et la nécropole de Baouit (notes mises en œuvre et éditées par D. Bénazeth et M.-H. Rutschowscaya), MIFAO 111, Le Caire, Ifao, 1999.

HADJI-MINAGLOU 2009

Gisèle Hadji-Minaglou, « Baouît », in Laure Pantalacci, Sylvie Denoix (éd.), « Travaux de l'Institut français d'archéologie orientale en 2008-2009», BIFAO 109, 2009, p. 560-564.

HADJI-MINAGLOU 2010

Gisèle Hadji-Minaglou, « Baouît », in Béatrix Midant-Reynes, Sylvie Denoix (éd.), « Travaux de l'Institut français d'archéologie orientale en 2009-2010 », BIFAO 110, 2010, p. 370-373.

HADJI-MINAGLOU 2016

Gisèle Hadji-Minaglou, « Baouît », in Rapport d'activité 2015-2016, suppl. au BIFAO 116, 2016, p. 191-201.

HADJI-MINAGLOU 2017

Gisèle Hadji-Minaglou, « Baouît », in Rapport d'activité 2016-2017, suppl. au BIFAO 117, 2017, p. $189-200$. 
HADJI-MINAGLOU 2018

Gisèle Hadji-Minaglou, « Baouît », in Rapport d'activité 2017-2018, suppl. au BIFAO 118, 2018, p. 185-196.

HUBER 2013

Béatrice Huber, « Eine Tunika fürs Jenseits », in Antoine De Moor, Cäcilia Fluck, Petra Linscheid (éd.), Drawing the Threads Together: Textiles and Footwear of the 1st Millennium AD from EgyptProceedings of the 7th Conference of the Research Group "Textiles from the Nile Valley", Antwerp, 79 October 2011, Tielt, Lannoo, p. 12-21.

LANSING 1933

Ambrose Lansing, in Ambrose Lansing, William C. Hayes Jr., « The Egyptian Expedition: The Excavations at Lisht », BMMA 28/11, 1933, p. 4-38.

SMITH et al. 2011

Joyce Y. Smith, Kristin South, C. Wilfred Griggs, Giovanni Tata, « Jewelry and Accessories from two Christian Burials from Fag el Gamous Cemetery in Fayum Region, Egypt », in Antoine De Moor, Cäcilia Fluck k (éd.), Dress Accessories of the 1st Millennium AD from Egypt: Proceedings of the 6th Conference of the Research Group "Textiles from the Nile Valley”, Antwerp, 2-3 October 2009, Tielt, 2011, p. 205-219.

\section{NOTES}

1. Cf. HADJI-MINAGLOU 2018, p. 186-187.

2. Cf. HADJI-MINAGLOU 2016, p. 197-199.

3. Deux sondages pratiqués en 2009, l'un sous l'escalier situé dans l'espace latéral nord du sanctuaire, l'autre dans la nef sud, devant le diaconicon, avaient révélé l'existence de sépultures antérieures à l'église : cf. HADJI-MINAGLOU 2009, p. 574.

4. Cf. HADJi-MinAGLOU 2010, p. 371-372, fig. 42.

5. Cf. HADJI-MiNAGLOU 2016, p. 194, fig. 2.

6. Le mur a été démonté sur $15 \mathrm{~m}$ à partir de l'entrée; les $3 \mathrm{~m}$ supplémentaires pour atteindre l'angle sud-ouest de l'église ont été dégagés de l'intérieur en 2017. L'angle extérieur n'a pu être entièrement mis au jour en raison de la présence de deux banquettes, qui ont été laissées en place et n'ont été déposées que cette année (voir le plan publié dans HADJI-MINAGLOU 2016, p. 191).

7. Cf. HADJI-MINAGLOU 2016, p. 197.

8. Cf. HADJI-MINAGLOU 2017, p. 199.

9. Les individus étudiés par Roberta Cortopassi en 2017 ont montré que, sous les fibres de palmier, les visages étaient protégés par un tissu placé en contact direct avec la tête : un résumé de l'examen des dépouilles mises au jour en 2016 et 2017 sera publié dans les actes des journées d'étude « Baouît (2008-2018) : panorama et perspectives. Rencontre de l'archéologie et des textes ", organisées en juin 2018 à Paris (cf. HADJI-MINAGLOU 2018, p. 196). Il en sera de même pour l'étude anthropologique effectuée par P. Bailet. Pour les corps découverts en 2019, seule l'étude anthropologique a été menée, celle des tissus le sera lors de la prochaine campagne. 
10. Une bouteille en verre avait été placée entre les jambes d'un individu (B11) dégagé en 2016 (cf. HADJI-MINAGLOU 2016, p. 198).

11. Cf. supra, n. 3.

12. Cf. supra, n. 6.

13. Cf. CLÉDAT 1999, p. 187-189 et p. 195-196, ph. 167-171. Ces fragments de stèle sont aujourd'hui conservés au musée du Périgord à Périgueux (inv. 2423 à 2428).

14. Cf. HADJI-MINAGLOU 2016, p. 198, fig. 7 et p. 199.

15. D'autres inhumations ont été repérées à proximité.

16. Cf. SмIтн et al. 2011, p. 214, fig. 7 et p. 215.

17. Cf. HUBER 2013, p. 12, fig. 3 et p. 14.

18. Cf. LANSING 1933, p. 25 et p. 29, fig. 36-37.

19. Cf. HADJI-MINAGLOU 2009, p. 563, fig. 23.

20. Cf. supra, n. 3.

21. Cf. HADJI-MINAGLOU 2009, p. 564.

22. Dans la pièce conservée contre le mur sud de l'église.

23. Cf. HADJI-MINAGLOU 2018, p. 195.

\section{INDEX}

Année de l'opération : 2019

Thèmes : IFAO

sujets https://ark.frantiq.fr/ark:/26678/pcrtwE5STw4SDe, https://ark.frantiq.fr/ark:/26678/ pcrtoV7zLJpAfX, https://ark.frantiq.fr/ark:/26678/pcrtd1J2aYQ6GE, https://ark.frantiq.fr/ark:/ 26678/pcrt4r4BzEK12w, https://ark.frantiq.fr/ark:/26678/pcrtrZlKwOpIJt, https://ark.frantiq.fr/ ark:/26678/pcrt6FJgRdSUXx, https://ark.frantiq.fr/ark:/26678/pcrtXnnrd43yND, https:// ark.frantiq.fr/ark:/26678/pcrtmGlRAoyFJ2, https://ark.frantiq.fr/ark:/26678/pcrtBXAyagmXvh lieux https://ark.frantiq.fr/ark:/26678/pcrtVaA82gFQn7

\section{AUTEURS}

\section{GISĖLE HADJI-MINAGLOU}

Archéologue-architecte, Ifao 